\title{
Methods for Measuring and Compensating Ball Screw Error on Multi-mode Industrial CT Scanning Platform
}

\author{
Yujie Zhang ${ }^{1, a}$, Shangfeng $\operatorname{Pan}^{1, b}$ and Jian Deng ${ }^{1, c}$ \\ ${ }^{1}$ Department of Mechanical Engineering, Tsinghua University, Beijing 100084, China \\ azhang-yj14@mails.tsinghua.edu.cn, bpansf@mail.tsinghua.edu.cn, \\ cdeng-j16@mails.tsinghua.edu.cn
}

Keywords: Positioning accuracy, Ball screw, Lead error, Backlash, Laser interferometer Abstract. Precision of ball screw had a great impact on the positioning accuracy of NC positioning platform. The lead error and backlash of ball screw, which could be accurately measured by laser interferometer, were two key factors affecting the positioning accuracy of NC positioning platform. By setting the compensation data in the numerical control system, significant improvement of the positioning accuracy of $\mathrm{NC}$ positioning platform could be achieved. A method for measuring lead error and backlash of ball screw by laser interferometer was introduced and another method for calculating compensation data basing on the results of measurement was putted forward. Experiments were carried out on a multi-mode industrial CT scanning platform to verify these two methods. And the positioning accuracy of multi-mode industrial CT scanning platform was advanced obviously by compensating ball screw lead error and backlash with the compensation data measuring by laser interferometer.

\section{Introduction}

In the semi-closed loop NC positioning platform, the positioning accuracy depends on the accuracy of ball screw. Screw error could be divided into load error, lead error and thermal error which affect the positioning accuracy of NC positioning platform obviously [1]. Load error was caused by the external force that applied to the screw, lead error was brought about by manufacturing precision and long-term friction wear, and thermal error was incited by the high temperature during operation. To heavy work load NC positioning platform, such as CNC lathes and CNC milling machine, thermal error and lead error play the major role in affecting the positioning accuracy, while to light work load NC positioning platform, including measuring instruments and laboratory apparatus, only lead error affected the positioning accuracy significantly. Meanwhile backlash led significant effects on the positioning accuracy in positioning platform which needs to reciprocate. Although the backlash could be reduced by mechanical structure, it could not be totally eliminated. A method for measuring lead error and backlash of ball screw by laser interferometer was introduced and another method for calculating compensation data was carried out in this paper. The methods were verified on a multi-mode industrial CT platform.

\section{A Method for Measuring Positioning Error of Multi-mode Industrial CT Scanning Platform}

The laser beam entered in the spectroscope as shown in Fig. 1, and was split into two beams. A laser beam called the reference beam was guided to a mirror mounted on the interferometer, while the other laser beam called measuring beam passed through the spectroscope and reached the second mirror. Then, two laser beams were reflected back to the beam interferometer, where they were re-combined and back into the laser transmitter. The detector in the laser transmitter monitored interference fringes changes while the second mirror moving [2]. During the linear measurement process, a mirror remained stationary, while the other mirror moved along the linear direction. By monitoring the changes of interference fringes, the precise moving distance could be calculated. 


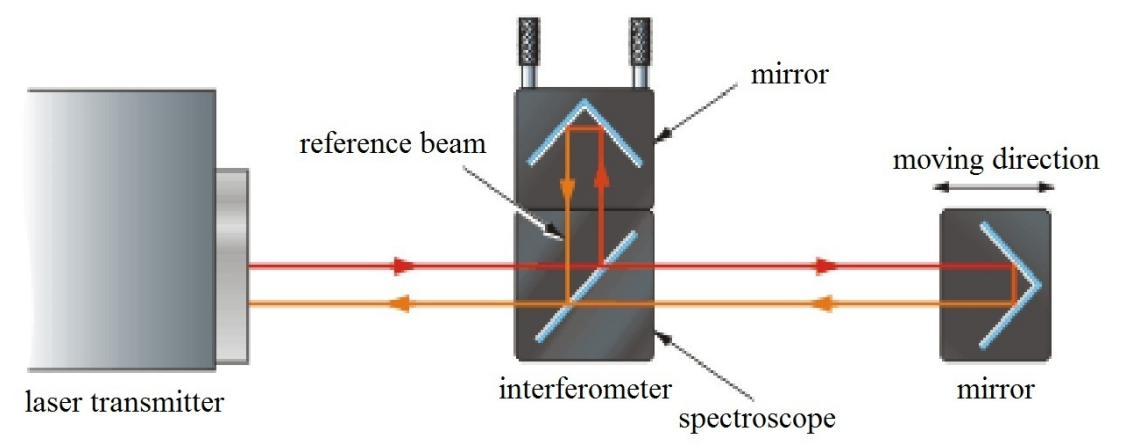

Fig. 1 The measuring principle of laser interferometer

Multi-mode industrial CT scanning platform was composed by the three movement axes, called $\mathrm{X}$-axes, $\mathrm{Y}$-axes and $\mathrm{Z}$-axes, as shown in Fig. 2. $\mathrm{X}$-axes and $\mathrm{Y}$-axes can drive the platform in horizontal direction, Z-axes can drive the platform in vertical direction. Renishaw XL30 laser interferometer measuring system was applied in the measuring experiment. The measuring system consisted of XL laser transmitter, interferometer, mirror and environmental compensation unit. The measurement precision of moving distance could be less than $1 u \mathrm{~m}$ and the resolution of moving distance could achieve less than $0.01 \mathrm{um} \mathrm{[3].} \mathrm{The} \mathrm{mirror} \mathrm{was} \mathrm{fixed} \mathrm{on} \mathrm{the} \mathrm{X}$ table by a magnetic seat, the laser transmitter was mounted on a tripod head and the interferometer was fixed on the base platform. The mirror, the laser transmitter and the interferometer should be in the same line meanwhile the parallel degree requirements of laser interferometer system should be meted. The experimental measurement site was showed in Fig. 3.

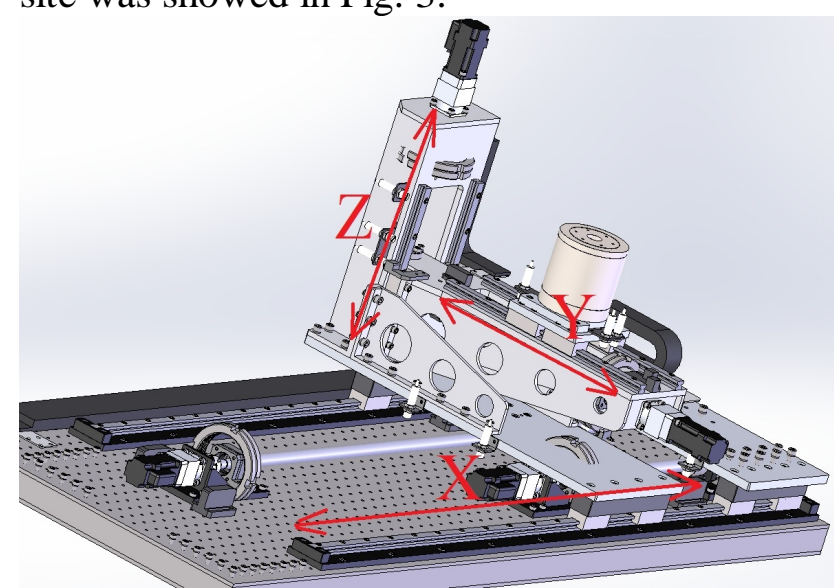

Fig. 2 3D model of industrial CT

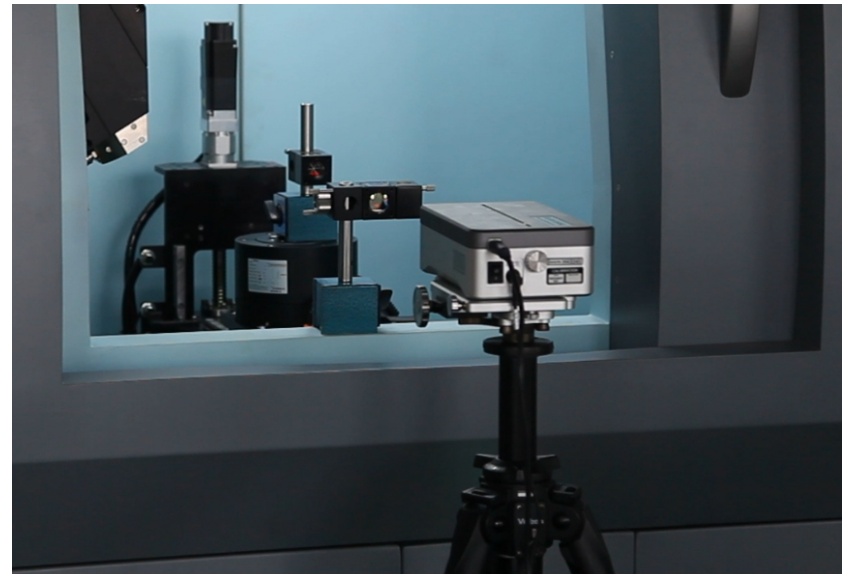

Fig. 3 Measurement system

\section{The Measurement Results of Positioning Accuracy without Compensation Data}

Precision-grade ball screws were selected to make up the multi-mode industrial CT scanning platform. In the initial state, there was no compensation data in the $\mathrm{NC}$ system. The $\mathrm{X}$-axis measurement results were described as an example. The travel of X-axis was $687.87 \mathrm{~mm}$ and the standard value of lead was $5 \mathrm{~mm} .11$ points were selected to measure the positioning accuracy of $\mathrm{X}$-axis, and each point measured 5 times in both positive and negative directions. The average positioning error were shown in Fig. 4. According to ISO 230-2 2014 [4], the positioning accuracy was calculated by below given equations from Eq. 1 to Eq. 5, the final calculation of the positioning accuracy was $0.0436 \mathrm{~mm}$.

Mean unidirectional positioning deviation at a position

$$
\overline{X_{i}} \uparrow=\frac{1}{n} \sum_{j=1}^{n} X_{i j} \uparrow
$$

and 


$$
\overline{X_{i}} \downarrow=\frac{1}{n} \sum_{j=1}^{n} X_{i j} \downarrow
$$

Estimator for the unidirectional axis positioning repeatability at a position

$S_{i} \uparrow=\sqrt{\frac{1}{n-1} \sum_{j=1}^{n}\left(X_{i j} \uparrow-\bar{X}_{i} \uparrow\right)^{2}}$

and

$S_{i} \downarrow=\sqrt{\frac{1}{n-1} \sum_{j=1}^{n}\left(X_{i j} \downarrow-\bar{X}_{i} \downarrow\right)^{2}}$

Bi-directional positioning accuracy of an axis

$A=\max \left[\overline{X_{i}} \uparrow+2 S_{i} \uparrow ; \overline{X_{i}} \downarrow+2 S_{i} \downarrow\right]-\min \left[\overline{X_{i}} \uparrow-2 S_{i} \uparrow ; \overline{X_{i}} \downarrow-2 S_{i} \downarrow\right]$

The value $n$ reminds of the number of measurements, the value $i$ reminds of the number of point.

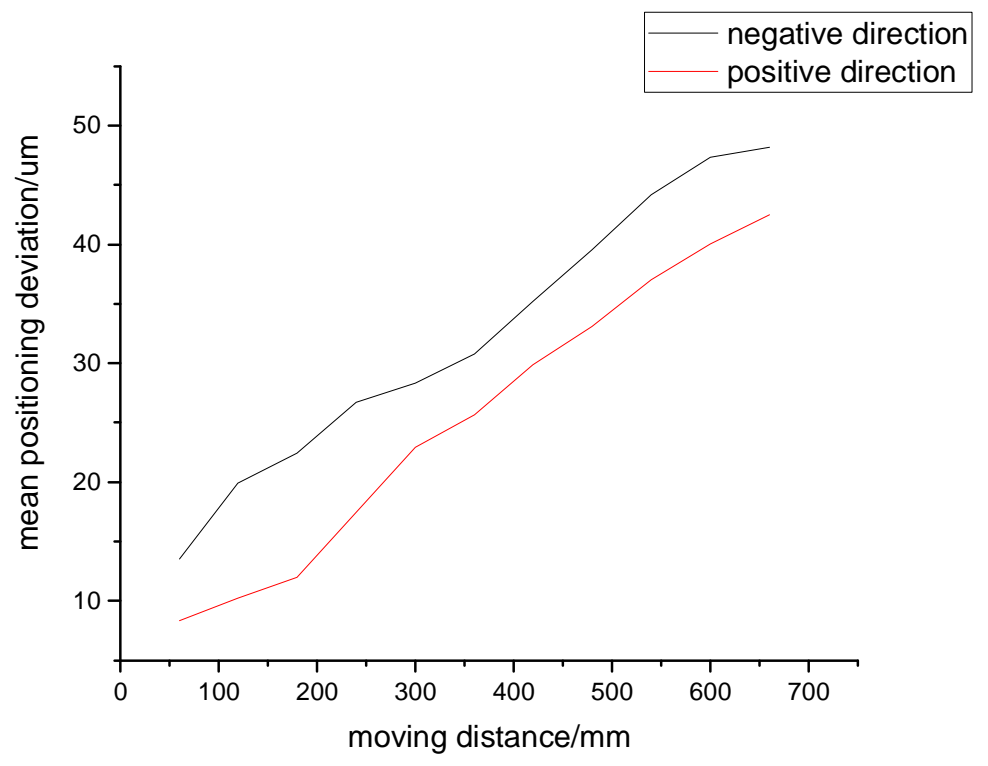

Fig. 4 Mean positioning deviation without compensation data

\section{Solving the Compensation Data Based on Optimization Method}

Considering the ball screw manufacturing errors and drive characteristics, the visual analysis for measurement results showed that the lead should be linear corrected and the backlash should be set to a fixed value. By setting the compensation data in the numerical control system, the positioning accuracy of multi-mode industrial CT scanning platform could be improved significantly. In accordance with the calculation method of positioning accuracy stipulated in ISO 230-2 2014, the bi-directional positioning accuracy of $\mathrm{X}$-axis was set as the objective function and exhaustive method was used in a certain range to solve optimum compensation data. The solving process was shown in Fig. 5 , and the relations of compensation data and positioning accuracy was shown in Fig. 6. The positioning error was minimal when the lead compensation value was set to $-0.0051 \mu \mathrm{m}$ and the backlash compensation value was set to $0.0071 \mu \mathrm{m}$. Theoretically, the positioning accuracy of X-axis could be less than $0.0079 \mathrm{~mm}$. 


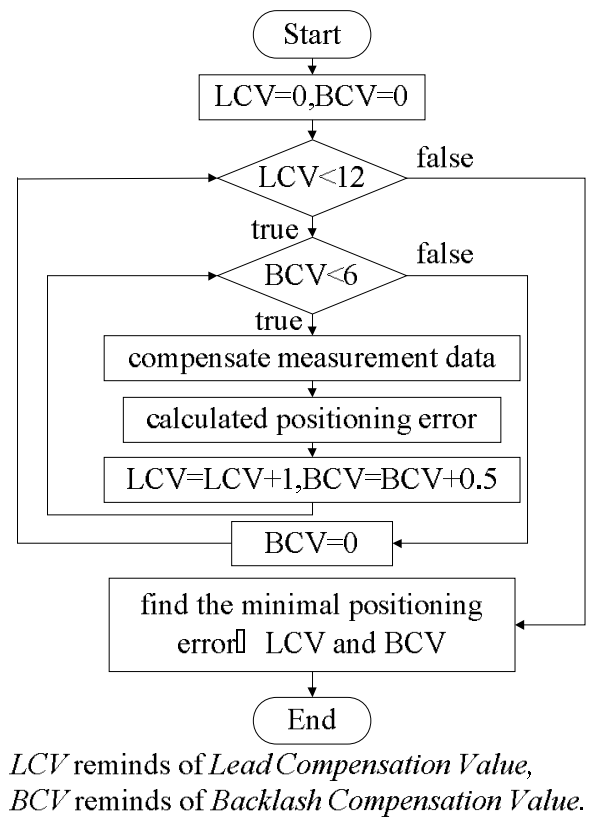

Fig. 5 The solving process of compensation data

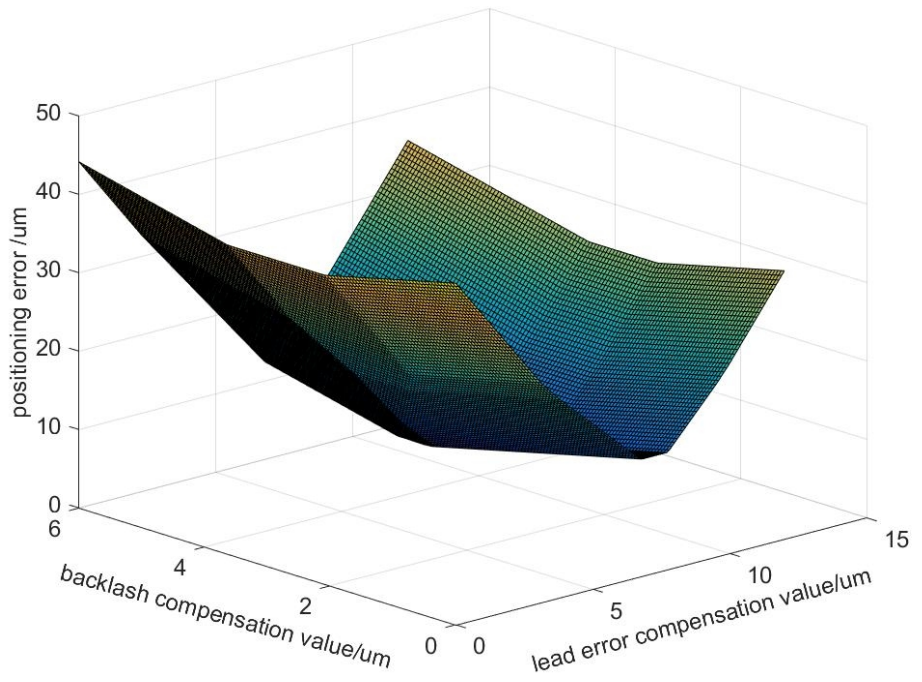

Fig. 6 The relations of compensation data and positioning error

\section{The Measurement Result of Positioning Accuracy with Compensation Data}

The compensation data mentioned above was set to numerical control system. Then, the positioning accuracy of $\mathrm{X}$-axis was measured using a laser interferometer. 11 points were selected to measure the positioning accuracy of $\mathrm{X}$-axis, and each point measured 5 times in both positive and negative directions. The average positioning error with compensation data were shown in Fig. 7. According to the calculation method of positioning accuracy stipulated in ISO 230-2 2014, the positioning accuracy of $\mathrm{X}$-axis was $0.014 \mathrm{~mm}$. 


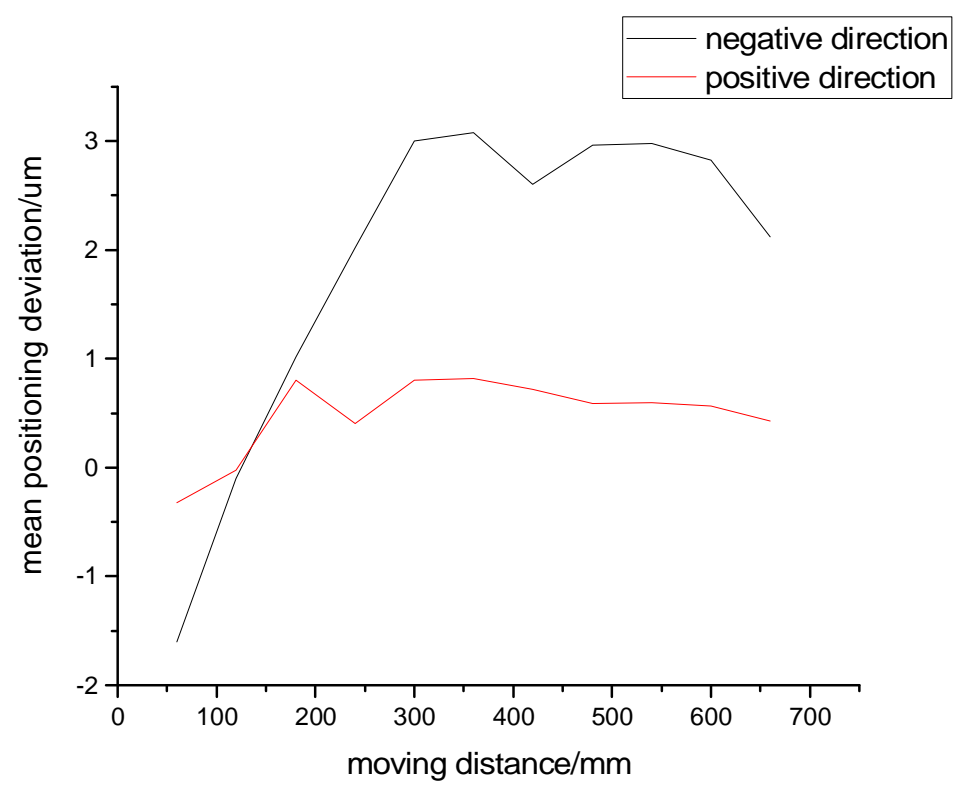

Fig. 7 Mean positioning deviation with compensation data

\section{Conclusions}

In summary, the positioning accuracy of multi-mode industrial CT scanning platform was advanced obviously by compensating screw lead and backlash with the compensation data measuring by laser interferometer. The screw lead and backlash would change because of screw abrasion in use, so the screw lead and backlash should be measured and compensated regularly. Due to the affection of temperature and other environmental factors, if much higher positioning accuracy was required, only using closed loop control system could meet the requirement.

\section{Acknowledgements}

This work was financially supported by the national key foundation for exploring scientific instrument of China (2014YQ240445).

\section{References}

[1] T. Satomi and S. Toba: Journal of Japan Society for Design Engineering Vol. 32-7 (1997), p. 23

[2] K. Zhang, Z.X. Cui, L.Y. Gai, P. Ge and D.G. Cai: Applied Mechanics \& Materials Vol. 130-134 (2011), p. 2316

[3] Information on http://www.renishaw.com

[4] ISO 230-2: 2014, Test Code for Machine Tools - Part 2: Determination of Accuracy and Repeatability of Positioning of Numerically Controlled Axes, an International Organization for Standardization (2014) 